\title{
Avanços no tratamento da esclerose múltipla através do anticorpo monoclonal Ocrelizumabe
}

\author{
Advances in the treatment of multiple sclerosis through monoclonal antibody \\ Ocrelizumab
}

Jonatas Gonçalves da Silva ${ }^{1}$ (D), Marina Ferri Pezzini² (D), Julia Poeta ${ }^{1}$ (C)

\begin{abstract}
RESUMO
Objetivos: Avaliar a efetividade e os riscos associados ao tratamento da esclerose múltipla com o anticorpo monoclonal Ocrelizumabe. Métodos: O estudo consiste em uma revisão da literatura objetivando a busca de artigos que evidenciassem de forma quantitativa os efeitos do fármaco Ocrelizumabe para as formas remitente-recorrente e primariamente progressiva da esclerose múltipla. A busca ocorreu nas bases de dados PubMed, BVS, Cochrane Library e ScienceDirect a partir de 2011. Os artigos foram pré-selecionados através do título e resumo para leitura integral e de acordo com os critérios de inclusão e exclusão. Resultados: No total, foram recuperados 743 artigos, sendo considerados elegíveis 23 estudos para análise integral e destes, 20 foram excluídos por não se enquadrarem nos desfechos propostos, sendo incluídos três estudos. Todos os estudos comprovaram uma alta eficácia de Ocrelizumabe livre de infecções oportunistas. Conclusão: apesar da natureza degenerativa da esclerose múltipla, os avanços tecnológicos na terapêutica através de fármacos como o Ocrelizumabe têm trazido resultados promissores, como a diminuição da taxa anual de surtos, do número de lesões por RM e da progressão da incapacidade. No entanto, devido a sua recente aprovação, não é possível avaliar os efeitos da utilização do anticorpo monoclonal a longo prazo.
\end{abstract}

Palavras-chave: Esclerose Múltipla; Anticorpo; Diagnóstico.

\begin{abstract}
Objective: Evaluating the effectiveness and risks associated with the treatment of multiple sclerosis with the monoclonal antibody Ocrelizumab. Methods: The study consists of a narrative review that aimed to search articles evidencing the effects of the Ocrelizumab drug for the recurrent and primary progressive forms of multiple sclerosis. The search was carried out in PubMed, BVS, Cochrane Library, and ScienceDirect databases from 2011. The articles were pre-selected through the title and abstract for full reading and included or excluded in the study according to predetermined criteria. Results: A total of 743 articles were retrieved, 23 of which were considered eligible for comprehensive analysis and 20 were excluded because they did not fit into the outcomes proposed. Three studies were included. All studies have demonstrated the high efficacy of Ocrelizumab free of opportunistic infections. Conclusion: Despite the degenerative nature of MS, technological advances in therapy through drugs such as Ocrelizumab bring promising results, such as reduced annual outbreak rates, the number of MRI injuries, and the progress of the disability. However, due to its recent approval, it is not possible to evaluate the long-term effects of monoclonal antibody use.
\end{abstract}

Keywords: Multiple Sclerosis; Antibody; Diagnosis.

1. Instituto de Ciências da Saúde, Centro Universitário Ritter dos Reis (UniRitter), Porto Alegre (RS), Brasil.

2. Latin American Cooperative Oncology Group (LACOG), Porto Alegre (RS), Brasil.

$\bowtie$ Julia Poeta. Coordenação da Biomedicina. Rua Orfanotrófio, 555 - Alto Teresópolis. CEP: 90840-440. Porto Alegre (RS), Brasil. julia_poeta@uniritter.edu.br | Recebido em: 26/08/2019 | Aprovado em: 08/11/2019 


\section{INTRODUÇÃO}

A Esclerose múltipla (EM) é uma desordem neurológica que já afeta cerca de 2,5 milhões de pessoas no mundo ${ }^{1,2}$. No Brasil, segundo dados do Ministério da Saúde, estimam-se que haja 35 mil portadores da doença, e, destes, 13 mil já estão em fase em tratamento. A prevalência nacional possui grande variabilidade por região, mas a média de brasileiros acometidos por EM é cerca de 15/100 mil habitantes ${ }^{3}$ acometendo frequentemente adultos jovens, entre 20 e 40 anos, e é mais comum em mulheres (proporção de dois para um) e em indivíduos de cor branca ${ }^{4}$.

A EM é uma doença neurodegenerativa crônica complexa, que tem como alvo o Sistema Nervoso Central (SNC) e acredita-se ser de natureza autoimune. É mediada por linfócitos autorreativos que atravessam a barreira hematoencefálica e entram no SNC, onde causam inflamação local que resulta em desmielinização, cicatrização gliótica e perda axonal ${ }^{2,4,5}$. Possui caráter multifatorial e, apesar dos fatores que acarretam esta reação ainda serem desconhecidos, acredita-se que uma desregulação imunológica desencadeada por diversos fatores ambientais e genéticos possam ser a causa da resposta imune. Ao se apresentarem num mesmo indivíduo, esses fatores originam uma disfunção do sistema imunológico, que consequentemente desencadeia uma ação autolesiva dirigida fundamentalmente contra a substância bran$\mathrm{ca}$, com perda de oligodendrócitos e mielina, resultando em um defeito na condução de impulsos nervosos e aparecimento de sintomas ${ }^{6,7}$. Essa autoimunidade induz uma reação inflamatória que atravessa a barreira hematoencefálica e secreta citocinas, estimulando a destruição da bainha de mielina ${ }^{8,9}$.

Na fase inicial da EM as manifestações clínicas são inespecíficas, observando-se fadiga, fraqueza muscular, disfunção motora e sensitiva, déficits cognitivos, problemas de visão, alterações esfincterianas, tremores e desequilíbrio. Em estágios mais avançados, a doença atua de forma disseminada, ocorrendo múltiplas lesões dispersas na grande região do encéfalo e da medula espinhal, dessa maneira, os sintomas são mais graves, ocasionando disfagia, comprometimento visual grave, paralisia total dos membros inferiores, além de comprometimento dos membros superiores, déficit cognitivo, demência e distúrbios emocionais ${ }^{6-11}$. Os sintomas variam le- vando em consideração a aleatoriedade dos focos de desmielinização, e a depressão é um dos sintomas que, frequentemente, encontra-se associado à EM, visto que trata-se de uma das doenças neurológicas de maior incapacitação física no adulto jovem? ${ }^{7}$.

A EM é marcada por surtos e remissões e a evolução clínica é utilizada para sua classificação em quatro tipos ${ }^{9-11}$ :

Remitente Recorrente (EM-RR);

Primariamente Progressiva (EM-PP);

Progressiva Recorrente (EM-PR);

Secundariamente Progressiva (EM-SP);

No diagnóstico, $85 \%$ dos indivíduos apresentam o tipo EM-RR caracterizada pela ocorrência de surtos súbitos, com duração de dias ou semanas, e posterior recuperação. A segunda forma mais comum com aproximadamente $10 \%$ dos casos é a EM-PP, a qual o paciente apresenta o surto, mas não se recupera totalmente, acumulando sequelas. A forma EM-SP é o resultado de uma evolução lenta e progressiva da EM-RR. Estima-se que oito a cada dez pessoas que foram diagnosticadas com o tipo EM-RR desenvolverão o tipo secundário progressivo. Ainda, a EM-PR é considerada a forma mais rara da doença, representando menos de $5 \%$ dos indivíduos diagnosticados, com sintomas de surtos sobrepostos com progressão evidente das incapacitações ${ }^{10-13}$.

O diagnóstico da doença é definido através dos critérios de McDonald, acompanhado da anamnese do paciente e exames laboratoriais. É efetuado um detalhado exame neurológico para a confirmação dos sintomas a fim de que sejam descartadas outras causas. Para confirmação do diagnóstico definitivo de EM os surtos precisam envolver diferentes áreas do SNC, prolongarem-se por mais de 24 horas e estarem separados por um período de pelo menos um mês. Os exames laboratoriais são utilizados como diferenciador de outras patologias com sintomas semelhantes ${ }^{14}$. Os critérios de McDonald são baseados na avaliação dos surtos da doença comparados com achados em exames de ressonância magnética $(R M)^{15}$. Uma atualização dos critérios de McDonald foi publicada em 2018, em que as bandas oligoclonais no líquido cefalorraquidiano (LCR) foram incluídas como critério diagnóstico, assim como lesões assintomáticas em RM ${ }^{16}$.

Em relação ao tratamento aplicado à EM, ainda não se é capaz de prevenir a ocorrência 
da doença, não há cura e tampouco um meio de restaurar a mielina danificada ou as funções perdidas, mas o enfoque principal do tratamento é o controle dos sintomas. Os tratamentos oferecidos são diversos e variam conforme a evolução e o estado do paciente. $O$ estadiamento da doença é definido através da Escala Expandida do Estado de Incapacidade (EEEI) ${ }^{16}$. Os tratamentos, em sua grande maioria, utilizam imunossupressores que auxiliam diminuindo a ativação do sistema imune e reduzindo os efeitos dos autoanticorpos - e, imunomoduladores - atuando como um "freio imunológico" na inflamação do SNC. Com a diminuição da atividade inflamatória, há redução dos surtos causados pela doença, promovendo meIhora na qualidade de vida do indivíduo ${ }^{17}$.

No Brasil, a Portaria Conjunta no 10, de 02 de abril de 2018, do Ministério da Saúde, dispõe de um protocolo de diretrizes terapêuticas para EM que auxilia os neurologistas do Sistema único de Saúde (SUS) na tomada de decisões. Este protocolo apresenta como primeira linha de tratamento os medicamentos: betainterferonas $1 \mathrm{a}$ ou $1 \mathrm{~b}$, acetato glatirâmer e teriflunomida. Em caso de falha terapêutica ou respostas subótimas é considerada a substituição por outro fármaco de primeira linha, ou os inclusos em segunda linha: fumarato de dimetila e fingolimode. Caso haja falha terapêutica na utilização de medicamentos da segunda linha é recomendado o uso de fingolimode, se não considerado na linha anterior. Atingindo a quarta e última linha de tratamento, o protocolo sugere a administração do anticorpo monoclonal Natalizumabe para o tratamento de EM-RR ${ }^{15}$. Alguns estudos já foram desenvolvidos demonstrando uma boa eficácia do Nataluzumabe ${ }^{18-19}$, entretanto, alguns pacientes apresentaram leucoencefalopatia multifocal progressiva (LMP), uma infecção oportunista fatal, causada pelo vírus John Cunningham (JC-vírus $)^{19}$, responsável por infectar os oligodendrócitos do SNC e destruí-los, causando incapacidade física grave ou até mesmo óbito ${ }^{20}$.

O Ocrelizumabe é um anticorpo monoclonal anti-CD20 recombinante humanizado, ou seja, que tem origem em outra espécie, mas possui suas sequências proteicas modificadas para se assemelhar aos anticorpos produzidos por humanos. Acredita-se que esta característica ajude a induzir menor imunogenicidade e reações à infusão ${ }^{20}$. O me- canismo de ação do fármaco baseia-se na ligação seletiva aos linfócitos B com o antígeno CD20 em sua superfície. Estes linfócitos desempenham papel fundamental na patogênese da EM, envolvendo-se na ativação dos linfócitos $T$, na secreção de citocinas e na produção de autoanticorpos, fatores estes que atacam a bainha de mielina dos neurônios. A destruição desta bainha resulta na dispersão dos impulsos nervosos e progressão da doença ${ }^{21,22}$.

A posologia do Ocrelizumabe consiste na infusão de uma dose intravenosa inicial de 300 mg seguida da mesma dose após duas semanas. A continuidade do tratamento é feita com doses subsequentes de $600 \mathrm{mg}$ a cada seis meses. Os eventos adversos que podem ser apresentados segundo a bula do produto são: reações à infusão, infecções do trato respiratório superior e inferior, depressão, dor nas costas, infecções associadas ao vírus do herpes, dor nas extremidades, infecções cutâneas, tosse, diarreia e edema periférico ${ }^{23}$.

Em 26 de fevereiro de 2017 foi aprovada, pela Agência Nacional de Vigilância Sanitária (ANVISA), a utilização do anticorpo monoclonal Ocrelizumabe para o tratamento da EM-RR e EM-PP EM ${ }^{21}$. O Ocrelizumabe apresenta respostas terapêuticas positivas e inovadoras, com poucos eventos adversos, além disso, não foram registrados relatos de LMP em ensaios que testaram a eficácia do fármaco $22-25$.

Estes resultados demonstraram a potencial utilização do anticorpo monoclonal Ocrelizumabe como alternativa ao Natalizumabe. Apesar de ter sido aprovado pela ANVISA para utilização, distribuição e comercialização, até o presente momento não houve fornecimento pelo SUS e por este motivo, não engloba o protocolo de diretrizes terapêuticas. Esta revisão foi realizada com o objetivo de avaliar a efetividade e segurança na utilização do Ocrelizumabe.

\section{MÉTODOS}

O estudo caracteriza-se por uma revisão sistemática de caráter quantitativo e exploratório. Considerando o objeto do estudo, os artigos referentes à temática abordada foram pesquisados no banco de dados das bibliotecas eletrônicas PubMed, Cochrane Library, BVS e ScienceDirect. 
Os resumos foram compilados e direcionados segundo os objetivos para a construção do artigo.

Foram incluídos na pesquisa artigos publicados de 2011 a 2019 que tinham como objetivo avaliar a eficácia do anticorpo monoclonal Ocrelizumabe no tratamento da EM-RR ou EM-PP. Também, deveriam descrever como desfecho a redução da taxa anual de surtos para EM-RR e da progressão de incapacidade para EM-PP, além de informar os possíveis eventos adversos causados por este fármaco. Artigos que não corresponderam com estes critérios foram excluídos, assim como os não disponíveis na íntegra.

Foram incluídos os estudos cuja pesquisa delineava ensaios clínicos randomizados (ECR's), coortes (longitudinais) ou de casos-controle. Também, deveriam conter de forma detalhada o protocolo de aplicabilidade.

A estratégia de busca foi baseada na construção de Strings que deveriam conter os seguintes termos: "multiple sclerosis relapsing remitting, multiple sclerosis, primary progressive e Ocrelizumab". Para a recuperação dos artigos no PubMed foi definida a seguinte String: "(((multiple sclerosis, relapsing remitting[MeSH Terms]) OR multiple sclerosis, primary progressive[MeSH Terms]) AND
Ocrelizumab)". Para busca na BVS a String definida foi: "(tw:(multiple sclerosis)) AND (tw:(relapsing remitting)) OR (tw: (primary progressive)) AND (tw:(ocrelizumab))". Estratégias semelhantes foram utilizadas nas bases de dados Cochrane Library e ScienceDirect. Para a maximização da recuperação de artigos fez se o uso de unitermos, que deveriam constar no título, no resumo ou nas palavras-chave das publicações.

\section{RESULTADOS}

No total, foram recuperados 743 artigos (PubMed: 38; Cochrane Library: 636; BVS: 44; ScienceDirect: 25), por meio das buscas eletrônicas. Inicialmente 101 artigos foram excluídos em virtude de duplicidade e 619 por não se enquadrarem nos critérios de inclusão mediante leitura do título e resumo. Assim, foram considerados elegíveis 23 estudos para análise integral do artigo. Destes, 20 foram excluídos por não se apresentarem nos desfechos propostos, sendo incluídos três estudos. Para melhor detalhamento dos estudos elegíveis, os dados estão dispostos na Tabela 1.

Tabela 1

Estudos clínicos utilizando o anticorpo monoclonal Ocrelizumabe

\begin{tabular}{|c|c|c|c|c|c|}
\hline Autor & Ano & População & Estudo & Local & Administração \\
\hline $\begin{array}{l}\text { Kappos } \\
\text { et al. }\end{array}$ & 2011 & $\begin{array}{l}220 \text { pacientes com } \\
\text { EM-RR }\end{array}$ & $\begin{array}{l}\text { Fase II, multicên- } \\
\text { trico, randomizado, } \\
\text { controlado por } \\
\text { placebo }\end{array}$ & $\begin{array}{l}79 \text { Centros em } \\
20 \text { países }\end{array}$ & $\begin{array}{l}1: 1: 1: 1 \\
\text { OCRE } 600 \mathrm{mg} \text { a cada } 24 \text { semanas } \\
\text { OCRE } 2000 \mathrm{mg} \text { a cada } 24 \text { semanas } \\
\text { Interferon beta-1a }(30 \mu \mathrm{g}) 2 \mathrm{x} / \\
\text { semana ou Placebo } 1 \mathrm{x} / \mathrm{semana}\end{array}$ \\
\hline $\begin{array}{l}\text { Montalban } \\
\text { et al. }\end{array}$ & 2017 & $\begin{array}{l}732 \text { pacientes com } \\
\text { EM-PP }\end{array}$ & $\begin{array}{l}\text { ORATORIO, Fase III, } \\
\text { multicêntrico, ran- } \\
\text { domizado, duplo-ce- } \\
\text { go, controlado por } \\
\text { placebo }\end{array}$ & $\begin{array}{l}219 \text { Centros em } \\
32 \text { países }\end{array}$ & $\begin{array}{l}2: 1 \\
\text { OCRE } 600 \text { mg via intravenosa ou } \\
\text { Placebo a cada } 24 \text { semanas } \\
\text { O duplo-cego foi administrado por } \\
\text { um mínimo de cinco doses ( } 120 \\
\text { semanas) até a ocorrência de } \\
\text { coorte de aproximadamente } 253 \\
\text { eventos de progressão de incapa- } \\
\text { cidade, confirmado por pelo menos } \\
12 \text { semanas }\end{array}$ \\
\hline $\begin{array}{l}\text { Hauser } \\
\text { et al. }\end{array}$ & 2017 & $\begin{array}{l}\text { OPERA I: } 821 \text { pa- } \\
\text { cientes com EM-RR } \\
\text { OPERA II: } 835 \text { pa- } \\
\text { cientes com EM-RR }\end{array}$ & $\begin{array}{l}\text { Ensaios idênticos de } \\
\text { fase III, randomiza- } \\
\text { dos, triplo-cegos }\end{array}$ & $\begin{array}{l}\text { OPERA I: } 141 \\
\text { Centros em } 32 \\
\text { países } \\
\text { OPERA II: } 166 \\
\text { Centros em } 24 \\
\text { países }\end{array}$ & $\begin{array}{l}\text { OCRE } 600 \mathrm{mg} \text { a cada } 24 \text { sema- } \\
\text { nas ou Interferon beta-1a } 44 \mu \mathrm{g} \\
\text { subcutâneo } 3 \times / \text { semana durante } 96 \\
\text { semanas }\end{array}$ \\
\hline
\end{tabular}


Tabela 1 (Continuação)

\begin{tabular}{|c|c|c|c|c|}
\hline Objetivo Primário & Resultados & Objetivos secundários & Resultados & Eventos adversos \\
\hline $\begin{array}{l}\text { Avaliar número } \\
\text { de lesões por } \\
\text { RM }\end{array}$ & $\begin{array}{l}\text { 89\% ( } 95 \% \text { IC 68-97) me- } \\
\text { nor no grupo OCRE } 600 \text { mg } \\
\text { em comparação ao placebo } \\
96 \% \text { ( } 95 \% \text { IC } 89-99 \text { ) } \\
\text { menor no grupo OCRE } \\
2000 \text { mg em comparação } \\
\text { ao placebo. } \\
\text { Os grupos OCRE } 600 \text { mg } \\
\text { e } 2000 \text { mg apresentam } \\
\text { melhores resultados em } \\
\text { comparação ao grupo } \\
\text { Interferon beta-1 }\end{array}$ & $\begin{array}{l}\text { Avaliar taxas de } \\
\text { recaída anualizada } \\
\text { durante } 24 \text { semanas. }\end{array}$ & $\begin{array}{l}0,13 \text { vs } 0,64 \\
80 \% \text { ( } 95 \% \text { IC } 45-99) \\
\text { menor no grupo OCRE } \\
600 \text { mg em compara- } \\
\text { ção ao Placebo } \\
0,17 \text { vs } 0,64 \\
73 \% \text { ( } 95 \% \text { IC } 29-97 \text { ) } \\
\text { menor no grupo de } \\
\text { OCRE } 2000 \text { mg em } \\
\text { comparação ao Placebo }\end{array}$ & $\begin{array}{l}\text { Reações à infusão } \\
\text { Relatos de infecção } \\
\text { semelhantes entre os } \\
\text { três grupos } \\
\text { Sem relatos de infec- } \\
\text { ções oportunistas }\end{array}$ \\
\hline $\begin{array}{l}\text { Avaliar a } \\
\text { progressão de } \\
\text { incapacidade }\end{array}$ & $\begin{array}{l}32,9 \% \text { com OCRE } 600 \mathrm{mg} \\
\text { vs } \\
39,3 \% \text { com Placebo } \\
(95 \% \text { IC } 0,59-0,98)\end{array}$ & $\begin{array}{l}\text { Avaliar a progres- } \\
\text { são da incapacidade } \\
\text { confirmada de } 24 \\
\text { semanas. } \\
\text { Caminhada crono- } \\
\text { metrada de } 25 \text { pés. }\end{array}$ & $\begin{array}{l}\text { Progressão da Incapa- } \\
\text { cidade em } 24 \text { semanas: } \\
29,6 \% \text { OCRE } 600 \text { mg } \\
\text { vs } \\
35,7 \% \text { placebo } \\
(95 \% \text { IC } 0,58-0,98) \\
\text { Caminhada cronome- } \\
\text { trada } 25 \text { pés: } 38,9 \% \\
\text { OCRE } 600 \text { mg vs } \\
55,1 \% \text { placebo } \\
(95 \% \text { IC } 1,6-51,5)\end{array}$ & $\begin{array}{l}\text { - Reações à infusão, } \\
\text { infecções do TRS, } \\
\text { herpes. } \\
\text { - Durante o trata- } \\
\text { mento, } 2,3 \% \text { dos } \\
\text { pacientes do grupo } \\
\text { OCRE apresentaram } \\
\text { alguma neoplasia. }\end{array}$ \\
\hline $\begin{array}{l}\text { Avaliar taxa } \\
\text { anual de surtos } \\
\text { em } 96 \text { semanas. }\end{array}$ & $\begin{array}{l}\text { OPERA I }(0,16 \text { vs } 0,29) \\
46 \% \text { menor com OCRE } \\
(p<0,001) \\
\text { OPERA II }(0,16 \times 0,29 ; \\
47 \% \text { menor com OCRE } \\
(p<0.001)\end{array}$ & $\begin{array}{l}\text { Progressão da In- } \\
\text { capacidade em } 12 \\
\text { semanas } \\
\text { Diminuição do nú- } \\
\text { mero de lesões }\end{array}$ & $\begin{array}{l}\text { OPERA I: } 94 \% \\
(p<0,001) \text { com OCRE } \\
600 \mathrm{mg} \\
\text { OPERA II: } 95 \% \\
(p<0,001) \text { com OCRE } \\
600 \mathrm{mg}\end{array}$ & $\begin{array}{l}\text { Em ambos os estu- } \\
\text { dos: } \\
\text { Reações relacionadas } \\
\text { à infusão, nasofarin- } \\
\text { gite, } \\
\text { infecção do TRS, ce- } \\
\text { faleia, infecção do TU }\end{array}$ \\
\hline
\end{tabular}

Fonte: próprio autor

Abreviaturas: OCRE, Ocrelizumabe; TRS, trato respiratório superior; TU, trato urinário; RM, ressonância magnética; EM-RR, esclerose múltipla remitente recorrente; EM PP, esclerose múltipla primeiramente progressiva

\section{DISCUSSÃO}

Nos estudos OPERA I e II, realizados por Hauser et al, a diminuição do número de lesões através da RM foi de $94 \%$ e $95 \%$, respectivamente, nos grupos tratados com Ocrelizumabe $600 \mathrm{mg}$ em comparação ao grupo Interferon Beta- ${ }^{24}$, assim como no estudo de Kappos et al, que apresentou uma redução nas lesões de $89 \%$ para o grupo Ocrelizumabe $600 \mathrm{mg}$ e $96 \%$ para o grupo Ocrelizumabe $2000 \mathrm{mg}$, ambos em relação ao grupo placebo ${ }^{22}$.

Em se tratando do risco relativo da progressão da incapacidade há também resultados semeIhantes. Nos ensaios clínicos OPERA I e II, houve redução de $40 \%$ nas 12 semanas de tratamento e que se manteve nas 24 semanas com Ocrelizumabe $600 \mathrm{mg}^{24}$. O estudo ORATORIO apresentou uma diminuição no risco relativo de progressão da incapacidade de $24 \%$, no entanto não é possível comparar com os estudos que avaliaram o Natalizumabe, pois este não é aprovado para o tratamento de EM-PP25.

Em se tratando da taxa anual de surtos em 96 semanas os resultados apresentam variabilidade considerando os comparadores do estudo (Interferon Beta-1a ou Placebo). Os resultados obtidos nos estudos OPERA I e II apresentaram uma redução de $46 \%$ e $47 \%$, respectivamente nos grupos que utilizaram Ocrelizumabe ${ }^{24}$. No estudo 
de Kappos et al. os autores avaliaram a taxa de recaída anual durante 24 semanas e os resultados obtidos foram redução de $80 \%$ com Ocrelizumabe $600 \mathrm{mg}$ e $73 \%$ para Ocrelizumabe 2000 mg 22 .

Considerando a eficácia e aceitabilidade, o Ocrelizumabe apresentou uma taxa relativamente mais alta e menores taxas de abandono em relação as outras terapias ${ }^{26}$.

Em geral, os resultados obtidos com o Ocrelizumabe assemelham-se aos achados nos primeiros ensaios que resultaram na aprovação do Natalizumabe para EM-RR, no entanto, não ocorreram relatos de infecções oportunistas pelo JC-vírus, responsável pelo encerramento antecipado do estudo $^{18,19}$. Este fato torna o Ocrelizumabe uma alternativa para pacientes que apresentarem resultado positivo para o vírus JC, entretanto, estudos com maior seguimento são necessários.

Essa revisão contribui para uma melhor compreensão da utilização do Ocrelizumabe em relação aos outros fármacos para o tratamento de EM. Os estudos demonstraram uma alta eficácia de Ocrelizumabe em ambas as formas da doença. Na EM-RR o anticorpo monoclonal foi capaz de reduzir significativamente o número de lesões, ou até mesmo evitar o surgimento destas, apresentando também uma redução na taxa anual de surtos. Na EM-PP, como primeiro medicamento a ser considerado de fato eficaz para esta variação da doença, o Ocrelizumabe teve resultados significativos em retardar a progressão da incapacidade dos indivíduos. Outra característica que tem agradado a quem faz uso do medicamento é a periodicidade de infusão, realizada a cada seis meses, não havendo a necessidade de infusões mensais, condição que se fazia necessária com o Natalizumabe. Os eventos adversos frequentemente relatados foram associados somente às primeiras infusões.

A partir da análise destes estudos, é possível destacar que apesar da natureza degenerativa da $E M$, os avanços tecnológicos na terapêutica através de fármacos como o Ocrelizumabe têm trazido resultados promissores, como a diminuição da taxa anual de surtos, do número de lesões por RM e da progressão da incapacidade. No entanto, devido a sua recente aprovação, não é possível avaliar os efeitos da utilização do anticorpo monoclonal a longo prazo. Por este motivo, se fazem necessários novos estudos para avaliar os efeitos provocados pelo uso contínuo do medicamento.

\section{REFERÊNCIAS}

1. Browne $P$, Chandraratna $D$, Angood C, Tremlett $H$, Baker C, Taylor BV, et al. Atlas of multiple sclerosis: A growing global problem with widespread inequity. Neurology. 2014;83(11):1022-4. doi: 10.1212/ WNL.0000000000000768

2. Dargahi $N$, Katsara $M$, Tselios $T$, Androutsou ME, Courten M, Matsoukas J, et al. Multiple sclerosis: Immunopathology and treatment update. Brain Sciences. 2017;7(7):78. doi: 10.3390/brainsci7070078

3. Ministério da Saúde (2014). Pesquisa com dados do DATASUS sobre mobilidade em pacientes com esclerose múltipla é um dos destaques do 150 Encontro Anual de Esclerose Múltipla BCTRIMS .Recuperado de: http:// datasus.saude.gov.br/noticias/atualizacoes/506-pesquisa-com-dados-do-datasus sobre-mobilidade-em-pacientes-com-esclerose-multipla-e-um-dos-destaques-do-15-encontro-anual-de-esclerose-multipla-bctrims-2014. Citado em 03 Dezmbro 2018.

4. DCNI (2016). Recomendações no Tratamento da Esclerose Múltipla e Neuromielite Óptica. Recuperado de :http://formsus.datasus.gov.br/novoimgarq/28121/4880987_312361. pdf. Citado em 03 Dezembro 2018.

5. Huang WJ, Chen WW, Zhang X. Multiple sclerosis: Pathology, diagnosis and treatments. Experimental and therapeutic Medicine. 2017;13(6):3163-66. doi: 10.3892/ etm.2017.4410

6. Syed YY. Ocrelizumab: A Review in Multiple Sclerosis. CNS Drugs. 2018;2(9):883-90. doi: 10.1007/s40263018-0568-7.

7. Yang $H$, Duchesneau E, Foster R, Guerin A, Ma E, Thomas NP. Cost effectiveness analysis of ocrelizumab versus subcutaneous interferon beta- $1^{a}$ for the treatment of relapsing multiple sclerosis. J Med Econ. 2017;20(10):105665. doi: $10.1080 / 13696998.2017 .1355310$.

8. Menge $T$, Dubey $D$, Warnke $C$, Hartung $H-P$, Stüve $O$, Menge $T$, et al. Ocrelizumab for the treatment of relapsing-remitting multiple sclerosis. Expert Rev Neurother. 2016;16(10):1131-9. doi: $10.1080 / 14737175.2016 .1227242$

9. Howard J, Trevick S, Younger DS. Epidemiology of Multiple Sclerosis. Neurologic Clinics. 2016;34(4):919-39. doi: $10.1016 /$ j.ncl.2016.06.016

10. Ciccarelli O, Barkhof F, Bodini B, Stefano N, Golay X, Nicolay K. Pathogenesis of multiple sclerosis: insights from molecular and metabolic imaging. Lancet Neurol. 2014;13(8):807-22. doi: 10.1016/S14744422(14)70101-2

11. Garg N, Smith TW. An update on immunopathogenesis, diagnosis, and treatment of multiple sclerosis. Brain Behav. 2015;5(9):1-13. doi:10.1002/brb3.362

12. Alves B, Angeloni R, Azzalis L, Pereira E, Perazzo F, Rosa $P$, et al. Esclerose múltipla: revisão dos principais tratamentos da doença. Saúde E Meio Ambiente: Revista Interdisciplinar. 2015;3(2):19-34. doi: https://doi. org/10.24302/sma.v3i2.542

13. Dendrou CA, Fugger L, Friese MA. Immunopathology of multiple sclerosis. Nature Reviews Immunology, 2015;15(9):545-58. doi: 10.1038/nri3871 
14. Filippini G. Ocrelizumab appears to reduce relapse and disability in multiple sclerosis but quality of evidence is moderate. Evid Based Med. 2017;22(6):215-6. doi: 10.1136/ebmed-2017-110721

15. Ministério da Saúde (2018). Protocolo Clínico e Diretrizes Terapêuticas da Esclerose Múltipla. Recuperado de: http://portalarquivos2.saude.gov.br/images/pdf/2018/ abril/09/PORTARIA- Citado em 03 Dezembro 2018.

16. Thompson AJ, Banwell BL, Barkhof F, Carroll WM, Coetzee T, Comi G. Diagnosis of multiple sclerosis: revisions of the McDonald criteria. The Lancet Neurology. 2018;17(2):162-73. doi: 10.1016/S1474-4422(17)30470-2.

17. Silva VM, Nascimento VMS. Esclerose Múltipla : Imunopatologia, Diagnóstico e Tratamento. Interfaces Científicas - Saúde e Ambient. 2014;2(3):81-90.

18. Polman $\mathrm{CH}, \mathrm{O}^{\prime}$ Connor $\mathrm{PW}$, Havrdova $\mathrm{E}$, Hutchinson $\mathrm{M}$, Kappos L, Miller DH. A randomized, placebo-controlled trial of natalizumab for relapsing multiple sclerosis. N Engl J Med. 2006;354:899-910. doi: 10.1056/NEJMoa044397

19. Rudick RA, Stuart WH, Calabresi PA, Confavreux C, Galetta SL, Radue EW. Natalizumab plus interferon beta-1a for relapsing multiple sclerosis. N Engl J Med. 2006;354:911-23. doi: 10.1056/NEJMoa044396

20. Fernández $O$, Fernández VE, Guerrero M. Tratamiento de la esclerosis múltiple. Med. 2015;11(77):4622-33. doi: https://doi.org/10.1016/j.med.2015.04.001
21. ANVISA (2017). Esclerose múltipla tem novo tratamento aprovado. Recuperado de: http://portal.anvisa. gov.br/rss/-/asset_publisher/Zk4q6UQCj9Pn/content/ id/4097328. Citado em 03 Dezembro 2018.

22. Kappos L, Li D, Calabresi PA, O'Connor P, Bar-Or A, Barkhof F. Ocrelizumab in relapsing-remitting multiple sclerosis: a phase 2, randomised, placebo-controlled, multicentre trial. Lancet. 2011;378(9805):1779-87. doi: 10.1016/S0140-6736(11)61649-8.

23. ROCHE (2018) Ocrevus $\AA$ (ocrelizumabe). Recuperado de: https://www.dialogoroche.com/content/dam/brasil/ bulas/o/ocrevus/Bula-OCREVUS-Profissional.pdf Citado em 03 Dezembro 2018.

24. Hauser SL, Bar-Or A, Comi G, Giovannoni G, Hartung $\mathrm{H}-\mathrm{P}$, Hemmer $\mathrm{B}$. Ocrelizumab versus Interferon Beta-1a in Relapsing Multiple Sclerosis. N Engl J. Med. 2017;376(3):221-34. doi: 10.1056/NEJMoa1601277.

25. Montalban X, Hauser SL, Kappos L, Arnold DL, Bar-Or A, Comi G. Ocrelizumab versus Placebo in Primary Progressive Multiple Sclerosis. N Engl J Med. 2017;376:209220. doi: $10.1056 /$ NEJMoa 1606468

26. Li H, Hu F, Zhang Y, Li K. Comparative efficacy and acceptability of disease-modifying therapies in patients with relapsing-remitting multiple sclerosis: a systematic review and network meta-analysis. J Neurol. 2019. doi:10.1007/s00415-019-09395-w 